\title{
Obtenção e caracterização de propelentes sólidos compósitos à base de PBLH, alumínio e perclorato de amônio com binders modificados com dióis de cadeia curta
}

\author{
Obtention and characterization of composite \\ solid propellants based on HTPB, aluminum \\ and ammonium perchlorate with modified \\ binders with short chain diols
}

\author{
${ }^{1}$ Divisão de Propulsão - APR/IAE/DCTA, Praça Marechal-do-ar Eduardo Gomes, n. 50, São José dos Campos, São \\ Paulo, Brasil. \\ ${ }^{2}$ Departamento de Química, Divisão de Ciências Fundamentais, ITA/DCTA, Praça Marechal-do-ar Eduardo Gomes, $\mathrm{n}$. \\ 50), São José dos Campos, São Paulo, Brasil. \\ e-mail: brunobccc@fab.mil.br
}

\begin{abstract}
RESUMO
O presente estudo avaliou diferentes formulações de propelentes sólidos à base de PBLH/AP/Al modificados por dióis de cadeia curta, 1,4-butanodiol e 1,2-propanodiol, em diferentes concentrações mássicas, com intuito de obter propriedades mecânicas diferenciadas sem negligenciar os demais requisitos impostos, tais como processabilidade e propriedades balísticas, destas mesmas formulações. Neste sentido, desenvolveu-se uma metodologia de cálculo das formulações, onde o processamento das mesmas foi realizado em reator de pequeno porte. Com a intenção de avaliar a influência do teor mássico de plastificante empregado, também foi processada uma formulação, de maior teor de 1,4-butanodiol, com maior quantidade de plastificante. Para tanto, avaliou-se o perfil de viscosidade dinâmica pós-processamentos, a evolução da propriedade mecânica da dureza durante a cura e realizaram-se ensaios mecânicos de tração e dureza finais. Realizaram-se também ensaios de determinação de massa específica, Raio-X das amostras e bomba calorimétrica. Ficou evidente que formulações modificadas com 1,4-butanodiol são promissoras. Não obstante, não foi possível alcançar no presente estudo todos os requisitos esperados em um produto final para emprego imediato.
\end{abstract}

Palavras-chave: propulsão sólida; propelente compósito; extensor de cadeia.

\begin{abstract}
The present study evaluated different formulations of solid propellants based on HTPB / AP / Al modified by the short chain diols, 1,4-butanediol and 1,2-propanediol at different mass concentrations. In this context, this was done in order to obtain differentiated mechanical properties without neglecting the other imposed requirements, such as processability and ballistic properties. In this sense, a methodology for calculating the formulations was developed and their processing was carried out in a small reactor. Moreover, with a view to evaluating the influence of the mass content of plasticizer used, the formulation containing a higher content of 1,4-butanediol was also processed employing a greater amount of plasticizer. The post-processing dynamic viscosity profile and the evolution of the hardness during curing were evaluated, and a mechanical characterization was performed. In addition, a specific mass determination test, X-rays of samples and a calorimetric bomb test were carried out. Hence, it became evident that the formulations modified with 1,4butanediol are promising. Nevertheless, it was not possible to achieve, in this study, all the requirements expected in a final product for immediate use.
\end{abstract}

Keywords: solid propulsion; composite propellant; chain extenders. 


\section{INTRODUÇÃO}

Os poliuretanos são provavelmente a classe de polímeros mais versátil devido a sua grande variedade de matérias-primas que podem ser utilizadas em sua obtenção [1]. Os pré-polímeros funcionalizados à base de polibutadieno são usados em propelente sólido compósito como combustível e percursor do binder de poliuretano. O binder, além de atuar como combustível, contribui para a integridade estrutural do propelente e confere parcialmente as suas propriedades mecânicas [2,3]. O motor foguete sólido (SRM) é utilizado em aplicações espaciais, no lançamento de satélites e cargas úteis, empregando o propelente sólido compósito como principal tipo de propulsores para foguetes [4-6]. Um dos principais conceitos de propulsão de foguete sólido é a propulsão química, em que um combustível e um oxidante, libera grande quantidade de gases em altas temperaturas e gera impulso ao motor do foguete, quando queimado [5-9]. Os propelentes podem ser classificados como líquidos, sólidos ou gasosos. Dentro da categoria de propelentes sólidos, se encontra a classe de propelentes sólidos compósitos [5-7, 9, 10]. Na obtenção de um propelente sólido compósito, cargas energéticas sólidas, plastificante e outros aditivos são incorporados ao binder, formado pela reação entre o pré-polímero à base de polibutadieno, sendo o mais conhecido o polibutadieno líquido hidroxilado (PBLH), e isocianatos di ou polifuncionais [3, 7]. O propelente sólido compósito consiste, então, em diversos componentes como oxidante, combustível metálico, binder, plastificante, agente de cura, estabilizantes e agentes reticuladores $[3,5,7]$. Os propelentes sólidos compósito devem atender a uma série de requisitos, pois devem ter propriedades mecânicas adequadas [3], para evitar a formação de fraturas e trincas, o que impediria que os motores em que são empregados cumpram sua missão final [11]. Os propelentes devem conter alta densidade energética e devem queimar de forma controlada. Além disso, a matriz polimérica, ou binder, deve ser capaz de absorver altas quantidades de cargas sólidas, sem impedir, no entanto, a realização dos processos de misturação e carregamento. Por outro lado, o binder tem efeito preponderante nas características mecânicas do propelente final [7, 12]. Os propelentes devem também ser capazes de manter sua integridade em ampla faixa de temperatura, em casos de rápida pressurização na ignição e devem ser resistentes às vibrações no transporte e à aceleração do bloco no lançamento do foguete [5,7]. Deste modo, as propriedades mecânicas são um dos parâmetros mais largamente analisados, pois são facilmente determinadas e por serem críticas em termos de integridade estrutural do grão carregado [13]. O método mais aplicado de caracterização das propriedades mecânicas, como o módulo de elasticidade, tensões máxima e na ruptura e os seus respectivos alongamentos, é o ensaio de resistência à tração $[14,15]$. Outros ensaios de caracterização, como o ensaio de determinação da dureza, também podem ser empregados na caracterização mecânica de propelentes [16]. A determinação da massa específica do propelente curado também é relevante em termos de caracterização do material compósito [17]. Durante o processamento também é relevante avaliar e otimizar as propriedades reológicas da pasta, amplamente estudadas através de determinação de viscosidade do propelente durante o carregamento [18-21].

Neste sentido, estudos de otimização de formulações de propelentes, em termos de propriedades balísticas e mecânicas, com objetivo de obter formulações cada vez melhores, são amplamente realizados [22-27].

O uso de dióis de cadeia curta na obtenção de binders de poliuretano se mostraram promissores em aplicações aeroespaciais $[28,29]$. Dióis são moléculas orgânicas que possuem dois grupos hidroxila ligados à cadeia carbônica. Estas hidroxilas, assim como as hidroxilas presentes nos pré-polímeros percursores de poliuretanos, reagem com grupos isocianatos, formando grupos uretano.

Apesar disso, estudos comparativos com caracterização de formulações completas de propelentes sólidos compósitos contendo dióis de cadeia curta ainda são incipientes.

Assim, este estudo objetivou avaliar formulações completas de propelentes sólidos compósitos empregando dois diferentes tipos de dióis de cadeia curta, 1,4-butanodiol (BDO) e 1,2-propanodiol, ou propilenoglicol, em diferentes quantidades. Para tanto, avaliou-se o perfil de viscosidade dinâmica pósprocessamentos, a evolução da propriedade mecânica da dureza durante a cura e realizaram-se ensaios mecânicos de tração e dureza finais. Realizaram-se também ensaios de determinação de massa específica, Raio-X das amostras e ensaio de bomba calorimétrica.

\section{MATERIAIS E MÉTODOS}

\subsection{Materiais}

Os materiais usados no presente estudo são apresentados na Tabela 1. 
Tabela 1: Materiais empregados no estudo.

\begin{tabular}{l|l|l|l}
\hline COMPONENTE & FABRICANTE & LOTE & FUNCIONALIDADE $\left(\mathbf{m m o l ~ g}^{-1}\right)$ \\
\hline Resina PBLH Liquiflex LIQ-P & Petroflex & 0700176 & 0,76 \\
\hline IPDI (Diisocianato de isoforona) & SNPE & $11 / 0016$ & 8,957 \\
\hline 1,4-butanodiol & Adrich & 50287 & 20,03 \\
\hline 1,2-propanodiol & Reagen & 881157 & 22,70 \\
\hline
\end{tabular}

\subsection{Cálculo Mássico das Formulações}

O estudo foi conduzido em um reator com capacidade de $420 \mathrm{~g}$ de propelente. A partir de uma formulação original já previamente estudada, com teor de binder, compreendendo pré-polímero e agente de cura, igual a $11,76 \%$, foi determinado que as massas de PBLH $\left(\mathrm{m}_{1}\right)$, IPDI $\left(\mathrm{m}_{2}\right)$, BDO ou propilenoglicol $\left(\mathrm{m}_{3}\right)$ somadas deveriam ser iguais a 49,3 $\mathrm{g}\left(\mathrm{m}_{4}\right)$, aproximadamente. Foram determinadas também as massas dos demais componentes da formulação, a partir da composição mássica de cada um. Além disso, foi proposto que a massa do 1,4-butanodiol ou do 1,2-propanodiol $\left(\mathrm{m}_{3}\right)$ fosse um percentual da massa de resina $\left(\mathrm{m}_{1}\right)$ utilizada na própria formulação, demonstrado matematicamente pelas Equações 1 e 2 .

$$
\begin{aligned}
& m_{1}+m_{2}+m_{3}=m_{4} \\
& m_{3}=x \cdot m_{1}
\end{aligned}
$$

Ao se ter duas equações, em princípio, e três incógnitas, o sistema ainda necessita de mais uma equação para que tenha única solução. A terceira equação é proveniente do parâmetro $\mathrm{R}$, definido como a relação da quantidade de grupos - NCO pela quantidade de grupos $-\mathrm{OH}$ presentes na formulação, em base molar, neste caso definido pela Equação 3. No presente estudo, adotou-se R igual a 0,9.

$$
R=\left(\frac{m_{2} \cdot I_{N C O I P D I}}{m_{1} \cdot I_{O H P B L H}+m_{3} \cdot I_{O H D I O L}}\right)
$$

Conforme Tabela 1, o $\mathrm{I}_{\text {NCOIPDI }}$ do IPDI empregado era igual a $8,957 \mathrm{mmol} \mathrm{g}^{-1}$. O I $\mathrm{I}_{\mathrm{OHPBLH}}$ era igual a 0,76 mmol g $\mathrm{m} \mathrm{I}_{\text {OHDIOL }}$ era 20,03 $\mathrm{mmol} \mathrm{g}^{-1}$ para o 1,4-butanodiol e 22,70 $\mathrm{mmol} \mathrm{g}^{-1}$ para o 1,2-propanodiol.

Ademais, optou-se por utilizar valores de $\mathrm{x}$ iguais a $0,0,05,0,10$ e 0,15 , sugeridos por estudos anteriores, de modo que foi possível a determinação das massas dos componentes do binder a serem empregadas nas formulações $\left(\mathrm{m}_{1}, \mathrm{~m}_{2}, \mathrm{~m}_{3}\right)$.

A Tabela 2 sugere um esquemático das formulações estudadas, bem como a nomenclatura utilizada no estudo. A título de exemplo, a formulação cuja massa de BDO seja $5 \%$ da massa de PBLH foi nomeada como "BDO $5 \%$ ".

Tabela 2: Formulações avaliadas e massas $\mathrm{m}_{1}, \mathrm{~m}_{2}$ e $\mathrm{m}_{3}$.

\begin{tabular}{c|c|c|c|c|c|c}
\hline FORMULAÇÃO & DIOL & $\mathbf{X}$ & $\mathbf{M}_{\mathbf{1}}$ & $\mathbf{M}_{\mathbf{2}}$ & $\mathbf{M}_{\mathbf{3}}$ & NOMENCLATURA \\
\hline 1 & - & 0,00 & 45,9 & 3,5 & 0,0 & BDO/PPG 0 \% \\
\hline 2 & BDO & 0,05 & 40,2 & 7,1 & 2,0 & BDO 5 \% \\
\hline 3 & BDO & 0,10 & 35,8 & 10,0 & 3,6 & BDO 10\% \\
\hline 4 & BDO & 0,15 & 32,3 & 12,2 & 4,8 & BDO 15\% \\
\hline 5 & PPG & 0,05 & 39,8 & 7,6 & 2,0 & PPG 5 \% \\
\hline 6 & PPG & 0,10 & 35,2 & 10,7 & 3,5 & PPG 10 \% \\
\hline 7 & PPG & 0,15 & 31,5 & 13,2 & 4,7 & PPG 15\% \\
\hline
\end{tabular}




\begin{tabular}{c|c|c|c|c|c|c}
\hline FORMULAÇÃO & DIOL & $\mathbf{X}$ & $\mathbf{M}_{\mathbf{1}}$ & $\mathbf{M}_{\mathbf{2}}$ & $\mathbf{M}_{\mathbf{3}}$ & NOMENCLATURA \\
\hline 8 & BDO & 0,15 & 32,3 & 12,2 & 4,8 & BDO $15 \%$ Modificada \\
\hline
\end{tabular}

Com exceção do plastificante DOA (dioctil adipato), e obviamente dos componentes do binder, todos os demais componentes tiveram as suas massas fixas em todas as formulações, com base na formulação original. A Tabela 3 elenca os componentes da formulação e os percentuais mássicos das formulações 1 a 7 e 8. O sistema empregado de perclorato de amônio foi bimodal, ou seja, com duas granulometrias médias distintas.

Tabela 3: Composição em base mássica das formulações estudadas.

\begin{tabular}{|c|c|c|c|}
\hline \multirow{2}{*}{ CLASSIFICAÇÃO } & \multirow{2}{*}{ COMPONENTE } & FORMULAÇÃO 1-7 & FORMULAÇÃO 8 \\
\hline & & $\%$ (EM MASSA) & $\%$ (EM MASSA) \\
\hline \multirow{3}{*}{ Aditivos } & Antioxidante & \multirow{3}{*}{2,25} & \multirow{3}{*}{5,35} \\
\hline & Plastificante DOA & & \\
\hline & Agente de ligação Amínico & & \\
\hline \multirow{3}{*}{ Sólidos } & Alumínio em pó & \multirow{3}{*}{86,00} & \multirow{3}{*}{83,30} \\
\hline & Óxido de Ferro III & & \\
\hline & Perclorato de Amônio & & \\
\hline \multirow{3}{*}{ Binder } & Resina PBLH LIQ-P & \multirow{3}{*}{11,75} & \multirow{3}{*}{11,39} \\
\hline & Diol (BDO ou PPG) & & \\
\hline & Agente de Cura IPDI & & \\
\hline
\end{tabular}

O DOA foi adicionado em maior quantidade em uma formulação chamada de BDO $15 \%$ Modificada com intuito de avaliar sua influência, a título de determinar a provável influência do plastificante em formulações que contenham dióis de cadeia curta. Em função da adição de DOA em maior quantidade, verifica-se uma redução do teor de sólidos da formulação e de binder na formulação 8.

A massa de DOA utilizada nessa formulação foi determinada pela Equação 4, de forma genérica, e pela Equação 5 já com os valores mássicos empregados no estudo.

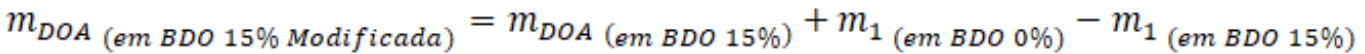

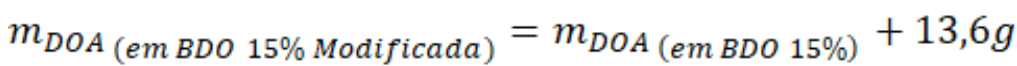

Esta equação foi sugerida com a intenção de compensar a diminuição da massa de pré-polímero, ou resina, no início do processo, que foi mais significativa nas formulações com maiores teores de dióis de cadeia curta.

\subsection{Processamento e cura do propelente}

No dia anterior a cada processo, foram pesadas as massas de plastificante DOA e de antioxidante utilizado. Misturou-se os dois, em um béquer, formando uma solução límpida de coloração amarelada. Esta solução foi armazenada em estufa a $55^{\circ} \mathrm{C}$, sob vácuo.

Do mesmo modo, a resina utilizada nos processos foi previamente seca em estufa também a vácuo a $55{ }^{\circ} \mathrm{C}$, onde foi mantida desde o dia anterior ao processamento. Por outro lado, os dióis foram secos em dessecador a vácuo, sem o emprego de aquecimento, pois observou-se considerável perda por evaporação dos mesmos quando aquecidos.

O perclorato de amônio foi separado em quantidade suficiente para os processos, sendo seco 
previamente a $80{ }^{\circ} \mathrm{C}$ por um período mínimo de 3 horas e desaglomerado, em seguida, com uma peneira. Após este procedimento, o material foi mantido em estufa também a $80{ }^{\circ} \mathrm{C}$ por no mínimo 12 horas e desaglomerado novamente pela passagem em peneira simples.

O alumínio em pó e o óxido de Ferro (III), utilizado como catalisador de queima, foram pesados em quantidade suficiente e secos em estufa a $100{ }^{\circ} \mathrm{C}$ por um período mínimo de 12 horas, para remoção de umidade.

O reator utilizado nos processamentos era do tipo horizontal, com controle de temperatura através de camisa alimentada por banho termostático, dotado de um sistema de vácuo. A temperatura de processo foi 50 ${ }^{\circ} \mathrm{C}$, ajustada pelo banho. As massas foram adicionadas gradativamente, a começar pela resina e pela solução de DOA e antioxidante. Em seguida, foram adicionados os sólidos e por último o diol de cadeia curta, finalizando as adições com o agente de cura. Entre a adição de um componente e outro da formulação adotou-se um tempo de mistura. Neste reator, não é possível variar a rotação, que foi mantida fixa durante todo processo. $\mathrm{O}$ vácuo era restabelecido sempre após a adição de um componente, mas a linha de vácuo era fechada logo em seguida. A intenção, neste caso, era remover todo ar presente no reator logo após sua abertura e adição de determinado componente. A adição de diol se deu praticamente no final do processo para evitar a perda deste por evaporação. O IPDI foi adicionado por último, pois a partir de sua adição iniciase a reação de cura, que aumenta a viscosidade do propelente. Após a adição de IPDI, a massa continuou a ser processada por um tempo $\mathrm{t}$ e foi carregada em um molde cúbico. Carregaram-se dispositivos para obtenção de discos cilíndricos para acompanhamento de dureza durante a cura, com intuito de avaliar seu progresso, duração e término. Cabe salientar que os tempos entre adições foram mantidos iguais em todos os processos.

A viscosidade foi acompanhada imediatamente após aos processos. Com intuito de comparar o perfil de viscosidade, importante parâmetro para avaliação da massa a ser carregada, mediu-se a viscosidade em viscosímetro Brookfield modelo DV-II+ Pro, a $50{ }^{\circ} \mathrm{C}$, spindle modelo SC4-25, na rotação de 0,3 rpm, no modo Stand-alone, a cada instante t minutos após a adição do IPDI no processamento. Obteve-se o gráfico viscosidade (cP) pelo tempo $\mathrm{t}(\mathrm{min})$, sendo t igual a zero no momento da adição de IPDI.

Os moldes cúbicos e os dispositivos cilíndricos carregados foram levados imediatamente para estufa de cura a $50{ }^{\circ} \mathrm{C}$, a pressão ambiente, e foram mantidos até que os valores de dureza dos discos obtidos através do dispositivo cilíndrico estabilizassem por dois dias consecutivos. A medição de dureza foi realizada pelo durômetro Instron modelo S1. Os valores de dureza (em ShA) pelo tempo de cura foram comparados nos distintos processamentos, com intuito de avaliar a influência dos dióis nos perfis de cura.

\subsection{Caracterização das amostras obtidas}

\subsubsection{Ensaios mecânicos - Tração e Dureza}

A massa de propelente curada foi cortada de modo a obter os corpos de prova para ensaios mecânicos. Adotou-se a norma técnica STANAG 4506 para realização dos ensaios de tração, empregando o equipamento INSTRON 3367, com velocidade de tração $50 \mathrm{~mm} \mathrm{~min}^{-1}$. Os ensaios foram realizados a $22{ }^{\circ} \mathrm{C}$, com o emprego de cinco corpos de prova, determinando a média dos valores de tensão máxima, tensão na ruptura e dos respectivos alongamentos. Também foram determinados os valores do módulo de elasticidade.

A dureza foi novamente medida, adotando-se a norma ASTM D2240 e utilizando o equipamento Durômetro Instron S1, realizando ao todo cinco medidas, determinando a dureza média e o desvio-padrão experimental (s).

\subsubsection{Ensaio de raio- $x$}

A partir dos corpos de prova rompidos no ensaio de tração, separou-se aleatoriamente três metades obtidas

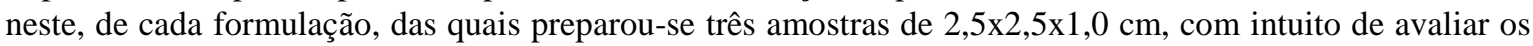
mesmos por meio de radiografias, com o objetivo de identificar possíveis distorções e maior incidência de vazios ou bolhas dentro da massa associadas a determinadas formulações.

\subsubsection{Determinação da massa específica do propelente}

As massas específicas dos propelentes curados foram determinadas experimentalmente utilizando o método hidrostático, com a intenção de avaliar a possível influência da presença de diol nos resultados finais de densidade do propelente. 
Amostras de propelente foram pesadas emersas e imersas em silicone utilizando o kit para determinação de densidade Mettler da balança semianalítica Mettler XPE205. A massa específica do silicone empregado foi $0,961 \mathrm{~g} \mathrm{~cm}^{-3}$.

\subsubsection{Ensaio de bomba calorimétrica}

Os calores de explosão e de combustão, segundo denominação encontrada em literatura [13], foram determinados, em triplicata, através do calorímetro modelo Parr 6200 Calorimeter, em atmosfera inerte $\left(\mathrm{N}_{2}\right)$ e oxidante $\left(\mathrm{O}_{2}\right)$, respectivamente. Massas de propelentes, próximas a 0,30 g, previamente pesadas, foram ignitadas para determinação do aumento da temperatura da água presente no banho do calorímetro, inicialmente a $25{ }^{\circ} \mathrm{C}$. A pressão inicial de operação foi de $30 \mathrm{~atm}$. Em virtude de a massa de propelente ser pequena, considera-se que a pressão do ensaio é constante. A energia aportada na queima do auxiliador de ignição e pelo fio de ignição são descontados, de modo que o valor final obtido representa apenas o calor liberado pela queima do propelente estudado. Para a determinação do calor de explosão empregou-se um auxiliador de ignição feito de $\mathrm{B}$ e $\mathrm{KNO}_{3}$ e para a determinação do calor de combustão utilizou-se ácido benzoico. O fio de ignição era composto por $\mathrm{Ni}$ e $\mathrm{Cd}$.

O ensaio de bomba calorimétrica é útil para determinação do conteúdo energético das amostras e avaliar a homogeneidade da massa, pois variações de calores gerados pelo mesmo material são resultado de diferenças de composição dentro da massa.

\section{RESULTADOS E DISCUSSÃO}

\subsection{Processamento e cura do propelente}

Optou-se por adicionar o diol após a adição de sólidos, antes da adição de IPDI, para evitar a formação de condensado no topo do reator, nas condições de processo. No entanto, em todos os processamentos, exceto da formulação 1, imediatamente após a adição dos dióis de cadeia curta, a massa teve uma inesperada abrupta mudança de reologia, com aumento visível de viscosidade, ainda que houvesse recém-adicionado um líquido de viscosidade baixa na mistura. Inferiu-se, portanto, que este comportamento era em função da presença desses dióis.

Porém, ao se adicionar o IPDI, em todos os casos, a massa imediatamente voltou a apresentar um aspecto de menor viscosidade, revertendo o quadro anteriormente descrito. Todos os processamentos que continham BDO na formulação puderam ser carregados sem maiores dificuldades, em escala laboratorial. Por outro lado, as massas finais das formulações 6 e 7, que continham 1,2-propanodiol em maior quantidade, tiveram viscosidade elevada no momento do carregamento, após o tempo de mistura final, com massa sem fluidez, inviabilizando o carregamento, em escala laboratorial.

Por último, foi realizado o processamento da formulação 8, com a intenção de avaliar a influência do teor de plastificante em uma formulação com alto teor de diol de cadeia curta, ao compará-la com a formulação 4.

A viscosidade foi acompanhada imediatamente após aos processos, com vistas a comparar o perfil de viscosidade, importante parâmetro para avaliação da massa a ser carregada. Os resultados de viscosidade pósprocessamento são apresentados na Figura 1. Cabe salientar que não foi possível acompanhar a viscosidade das formulações 6 e 7, pois ao término do processo a massa já não possuía fluência, inviabilizando as medições, nas circunstâncias experimentais. 


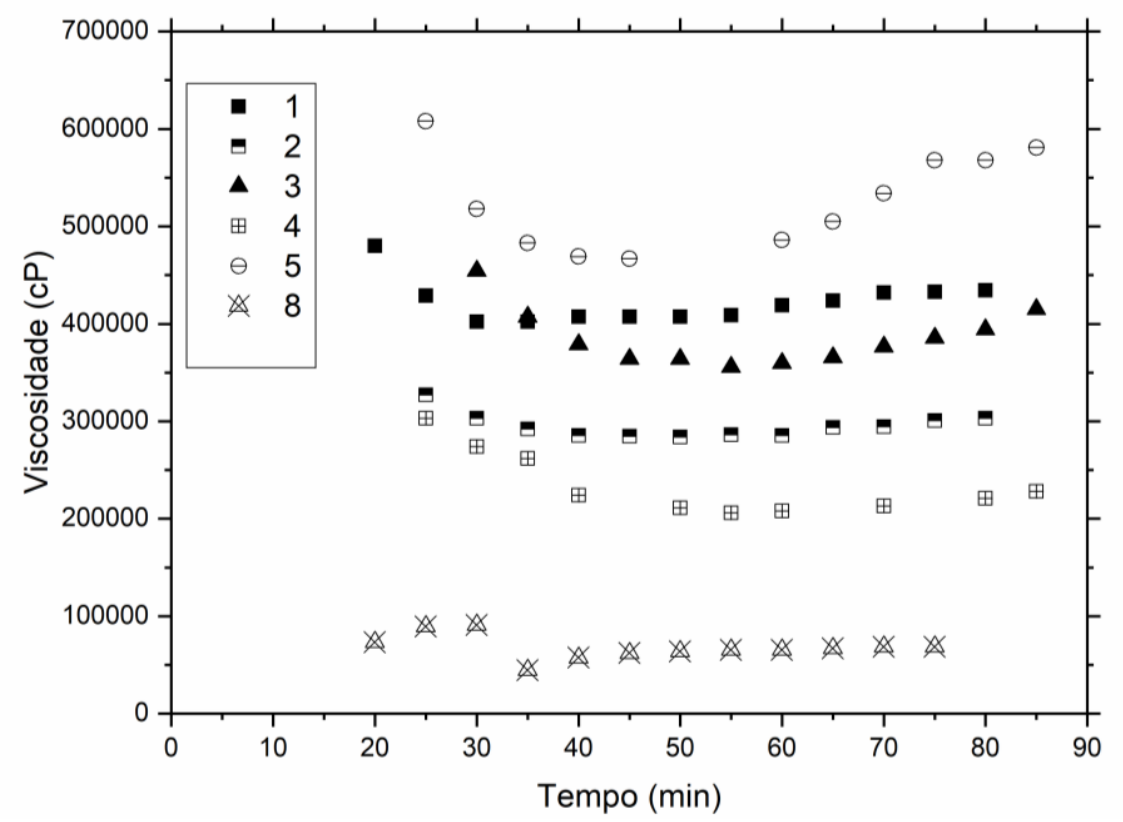

Figura 1: Evolução da viscosidade das misturas imediatamente após os processamentos.

A partir dos dados apresentados, e em função da impossibilidade de medição para as formulações 6 e 7, sugere-se que a adição de BDO diminui a viscosidade da massa final nos instantes iniciais pósprocessamento, visto que a viscosidade de todas as formulações com BDO, formulação 2, 3 e 4, apresentaram valores de viscosidade inferiores ao apresentado pela formulação de referência 1, sem diol.

Também se verificou que nas formulações com a presença de BDO não há um aumento considerável e rápido da viscosidade na massa final, o que facilitaria a operação de carregamento do propelente em escala industrial.

No entanto, a presença de propilenoglicol faz com que a viscosidade da massa aumente, se comparado com a formulação original, sendo maior quanto maior o teor de 1,2-propanodiol, inclusive pelo fato de não ter sido possível medi-la já a partir da formulação com x igual a 0,10 , devido à cura acelerada na presença de 1,2-propanodiol em altas concentrações.

Neste sentido, pode-se inferir que a presença do propilenoglicol, sendo um diol de cadeia menor que o BDO, e também, tendo em vista a maior proximidade na cadeia das hidroxilas reativas, resulta em uma velocidade de cura no início do processo maior, reduzindo consideravelmente o tempo disponível para o carregamento da massa.

Assim, sugere-se que o tempo de mistura pós-adição de IPDI deveria ter sido menor, ou deveria ter sido empregado maior teor de plastificante nos processamentos com propilenoglicol, para viabilizar o carregamento das formulações 6 e 7. No entanto, os tempos de mistura foram mantidos iguais em todas as formulações para permitir uma comparação mais fidedigna e não foram processadas formulações com 1,2propanodiol empregando maior teor de plastificante.

A queda na viscosidade, no início do acompanhamento, pode ser explicada, porém, pelo fato do BDO atuar como plastificante, digamos, no início, antes de reagir propriamente dito com o IPDI presente no meio.

Com relação à formulação 8 , a viscosidade final da massa foi muito baixa, o que pode ser prejudicial ao propelente final, pois poderia permitir a sedimentação dos sólidos presentes na mistura. Os valores de viscosidade da formulação 8 são amplamente inferiores aos das demais massas, sugerindo que a presença de DOA em teores mássicos na ordem de $5 \%$ para a formulação sugerida mostrou-se ser excessiva.

Os valores de dureza (em ShA) pelo tempo de cura foram comparados nos distintos processamentos, com intuito de avaliar a influência dos dióis nos perfis de cura. A Figura 2 apresenta os dados de dureza pelo tempo obtidos em todos os processamentos. 


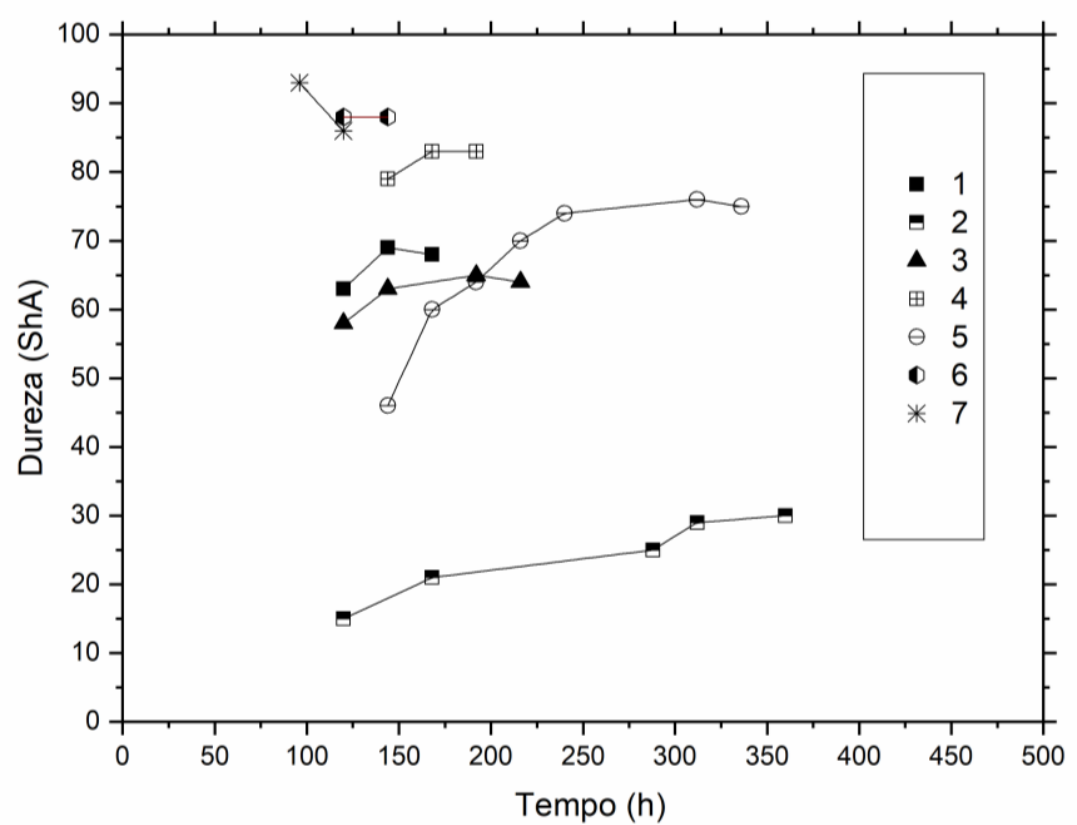

Figura 2: Evolução da dureza no tempo no acompanhamento do processo de cura dos propelentes.

Conforme já sugerido pelos perfis de viscosidade no início do processo, a cura para formulações com a presença de propilenoglicol, para teores elevados, foi muito mais acelerada, exemplificadas pelas formulações 6 e 7, cujos processos de cura finalizaram em menos de 150 horas. A presença de teor alto de $\mathrm{BDO}$ na formulação 4 também resultou em um processo de cura mais rápido, se comparado com as demais formulações que levavam BDO em menor quantidade.

No entanto, a presença de dióis de cadeia curta em teores baixos, para x igual a 0,05 e 0,10 no caso do $\mathrm{BDO}$, e para $\mathrm{x}$ igual a 0,05 no caso do propilenoglicol, resultou no retardo do processo de cura, pela maior quantidade de tempo necessário para estabilização da dureza. Isto se deve ao fato de os dióis de cadeia curta atuarem, de certa forma, como plastificante da resina presente, dificultando que a mesma reaja com o IPDI adicionado no meio. Esse efeito seria mais intenso quanto maior fosse a molécula do diol, haja visto que as formulações com a presença de BDO demoraram mais tempo que seus pares de mesmo teor na presença de propilenoglicol.

\subsection{Caracterização das amostras obtidas}

\subsubsection{Ensaio de dureza}

Os resultados dos ensaios de dureza finais das amostras são apresentados na Figura 3. 


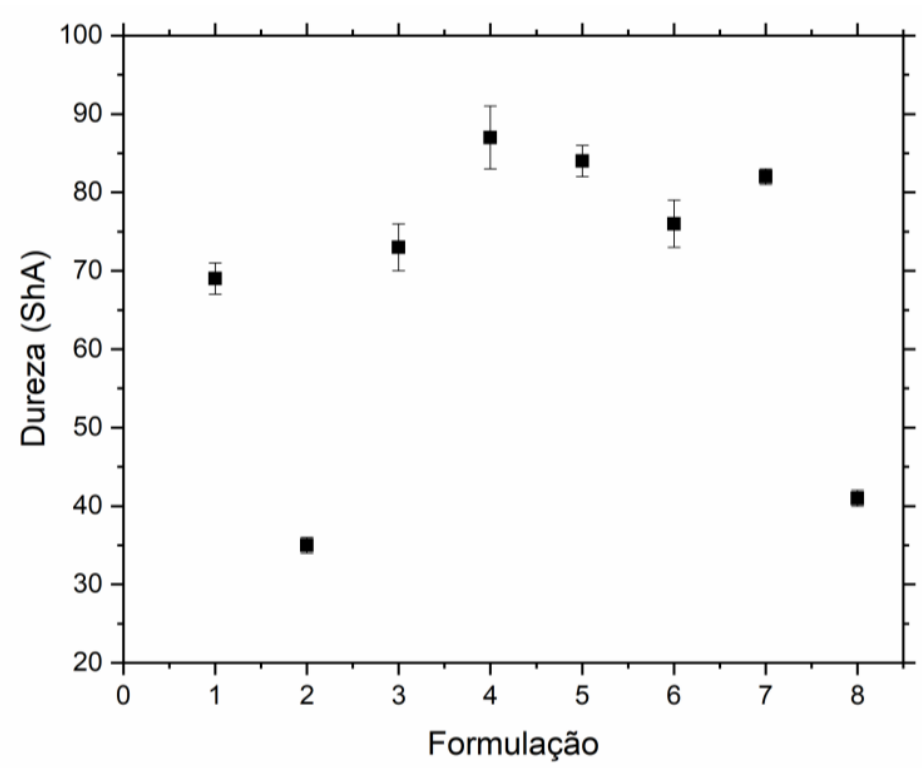

Figura 3: Resultado gráfico de dureza obtidos das amostras curadas.

Os resultados sugerem que, para o caso das formulações que continham BDO, houve uma redução considerável na dureza para o menor teor de BDO, para x igual a 0,05, em relação à formulação original. Podemos inferir que a presença de BDO em pequenas quantidades, como já havia sido sugerido no perfil de viscosidade durante a cura, impediu parcialmente a reação de cura entre a resina e o IPDI, diminuindo a reticulação e conferindo ao material menor dureza. No caso da formulação 3 , houve um restabelecimento da dureza inicial, em função da maior quantidade de ligações cruzadas, pela maior quantidade de ligações IPDIBDO, e pelo fato do BDO ter cadeia mais curta que a Resina PBLH. Do mesmo modo, a presença em altos teores de BDO acarretam grande quantidade de ligações IPDI-BDO, que tornam a estrutura muito reticulada, sugerido pelo alto valor de dureza, na ordem de $87 \mathrm{ShA}$ para a formulação 4 . Por último, a adição de DOA em maior percentual na formulação 8 , reduziu a dureza final, confirmando que o plastificante atua entre as moléculas, afastando os grupos da rede uretânica, conferindo à massa final menor dureza, com relação à formulação 4, que também continha BDO e possuía mesmo valor de x. Por outro lado, o valor de R igual a 0,9 não se mostrou adequado, por tornar o propelente mais quebradiço, para formulações com valores de $\mathrm{x}$ iguais ou superiores a 0,10 , em formulações com BDO, para o teor de sólidos empregado neste estudo. Assim, seriam recomendados valores inferiores de $\mathrm{R}$, para reduzir a dureza desses propelentes, ao conferir à formulação menor quantidade de reações entre os grupos $\mathrm{OH}$ e $\mathrm{NCO}$, dando maior mobilidade à matriz.

Com relação ao grupo de formulações com propilenoglicol, que inclui as formulações 5 , 6 e 7 , não foi possível carregar a caixa de maneira uniforme para as formulações 6 e 7, em virtude da alta viscosidade da massa no final do processamento. No entanto, a única massa que foi possível obter corpos de prova para ensaio mecânico, a formulação 5, apresentou dureza superior ao seu par, de mesmo x, com BDO. A dureza das formulações 6 e 7 são apresentadas apenas a título de informação, mas não devem entrar na análise pois foram obtidas a partir de corpos de prova inadequados, devido à dificuldade de realização do carregamento para estas duas misturas. Assim, sugere-se que o 1,2-propanodiol, por ter cadeia menor que o BDO, e as hidroxilas mais próximas, torna o propelente mais duro, ao comparar-se com propelentes à base de BDO.

\subsubsection{Ensaio de tração}

A Figura 4 apresenta os resultados gráficos obtidos nos ensaios de tração realizados no presente estudo. 


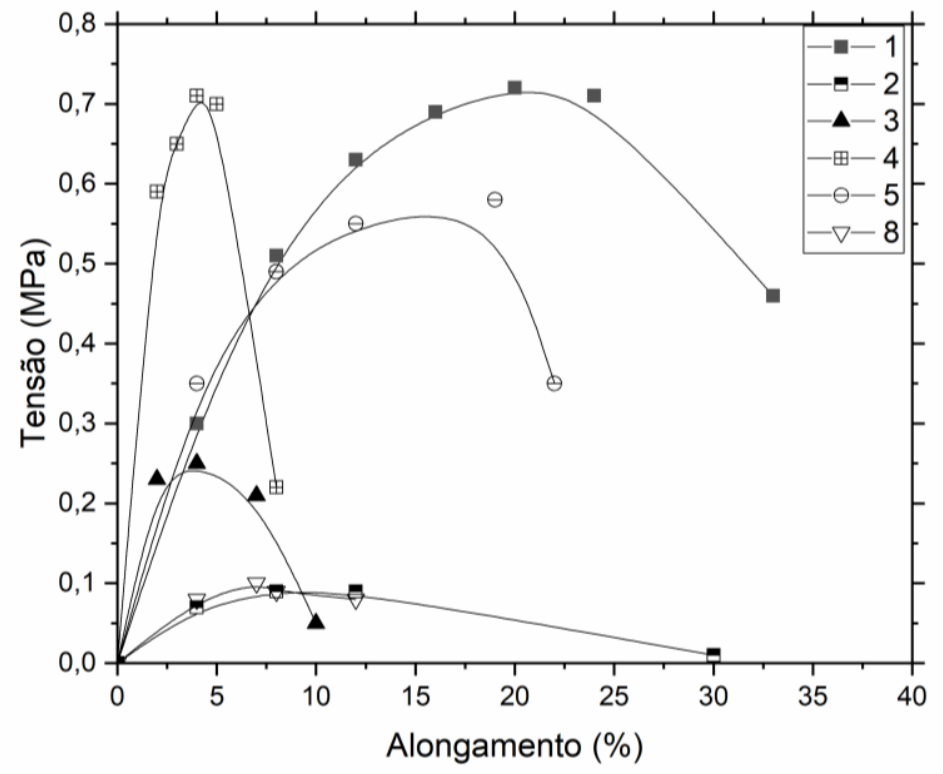

Figura 4: Gráfico de Tensão (MPa) versus Alongamento (\%) para as formulações de propelente curado.

Os resultados de tensão máxima, tensão na ruptura, alongamento na tensão máxima e na ruptura e Módulo de Young são apresentados na Tabela 4 e nas Figuras 5,6 e 7, a continuação.

Tabela 4: Dados obtidos nos ensaios de tração para as amostras de propelente curado.

\begin{tabular}{|c|c|c|c|c|c|c|c|c|c|c|}
\hline \multirow{2}{*}{ FORMULAÇÃO } & \multirow{2}{*}{$\begin{array}{c}E_{0} \\
(\mathrm{MPa})\end{array}$} & \multirow{2}{*}{$\begin{array}{c}\mathrm{s} \\
(\mathrm{MPa})\end{array}$} & \multicolumn{4}{|c|}{ ALONGAMENTO $\varepsilon(\%)$} & \multicolumn{4}{|c|}{ TENSÃO $\sigma$ (MPa) } \\
\hline & & & MÁX & $\mathbf{s}$ & RUPTURA & $\mathbf{s}$ & MÁX & $\mathbf{s}$ & RUPTURA & $\mathbf{s}$ \\
\hline 1 & 7,22 & 1,21 & 29 & 5 & 33 & 5 & 0,76 & 0,09 & 0,46 & 0,14 \\
\hline 2 & 1,08 & 0,13 & 12 & 3 & 30 & 6 & 0,09 & 0,09 & 0,01 & 0,01 \\
\hline 3 & 4,59 & 0,24 & 4 & 1 & 10 & 3 & 0,25 & 0,01 & 0,05 & 0,04 \\
\hline 4 & 13,47 & 1,56 & 5 & 3 & 8 & 3 & 0,70 & 0,11 & 0,22 & 0,06 \\
\hline 5 & 7,30 & 0,22 & 19 & 3 & 22 & 4 & 0,58 & 0,02 & 0,35 & 0,04 \\
\hline 8 & 1,94 & 0,10 & 7 & 1 & 14 & 1 & 0,10 & 0,01 & 0,06 & 0,01 \\
\hline
\end{tabular}




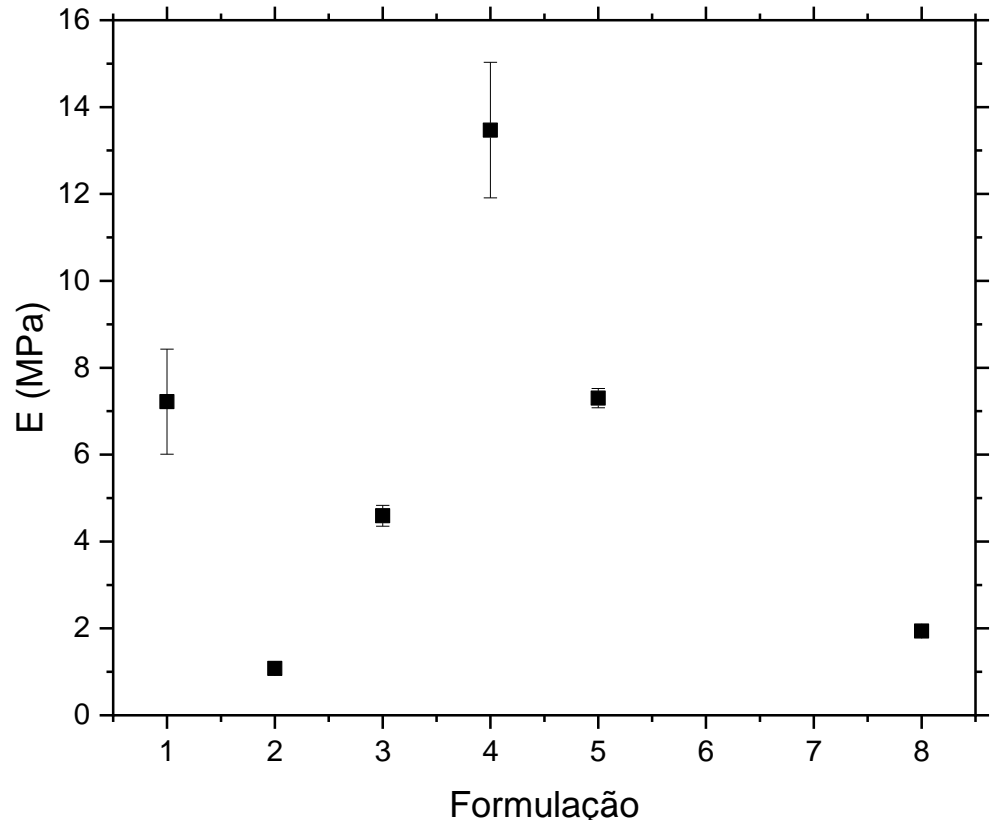

Figura 5: Módulo de Elasticidade (MPa) por formulação de propelente curado.

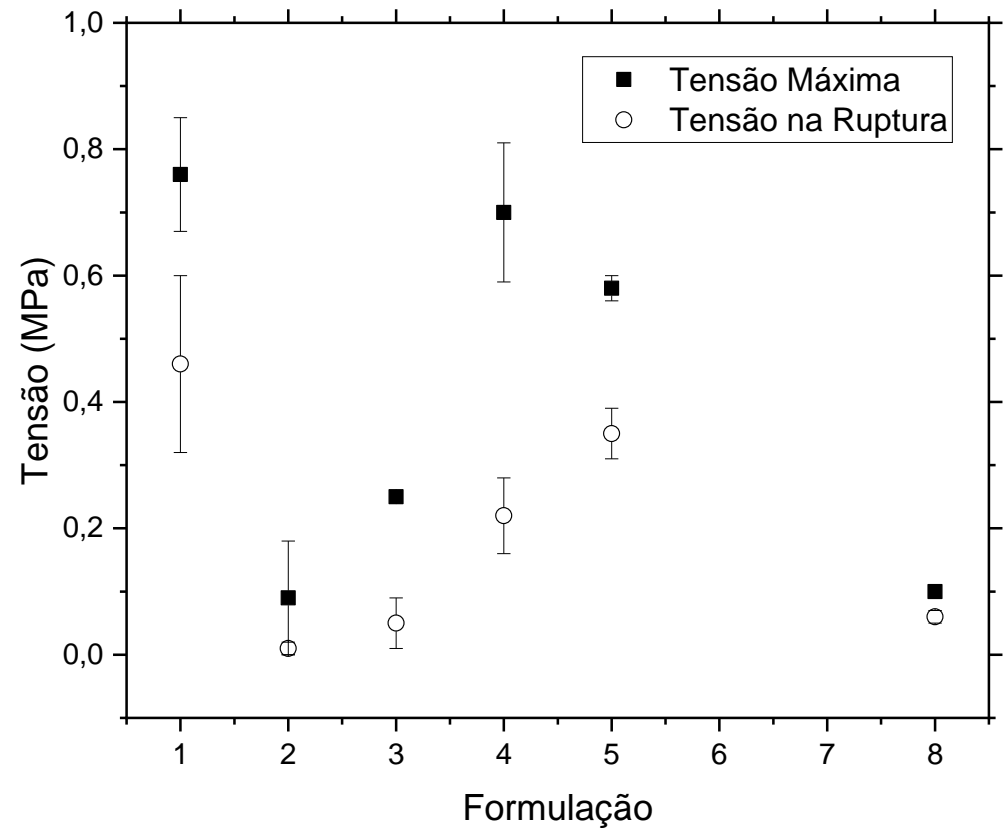

Figura 6: Tensão Máxima e na Ruptura (MPa) por formulação de propelente curado. 


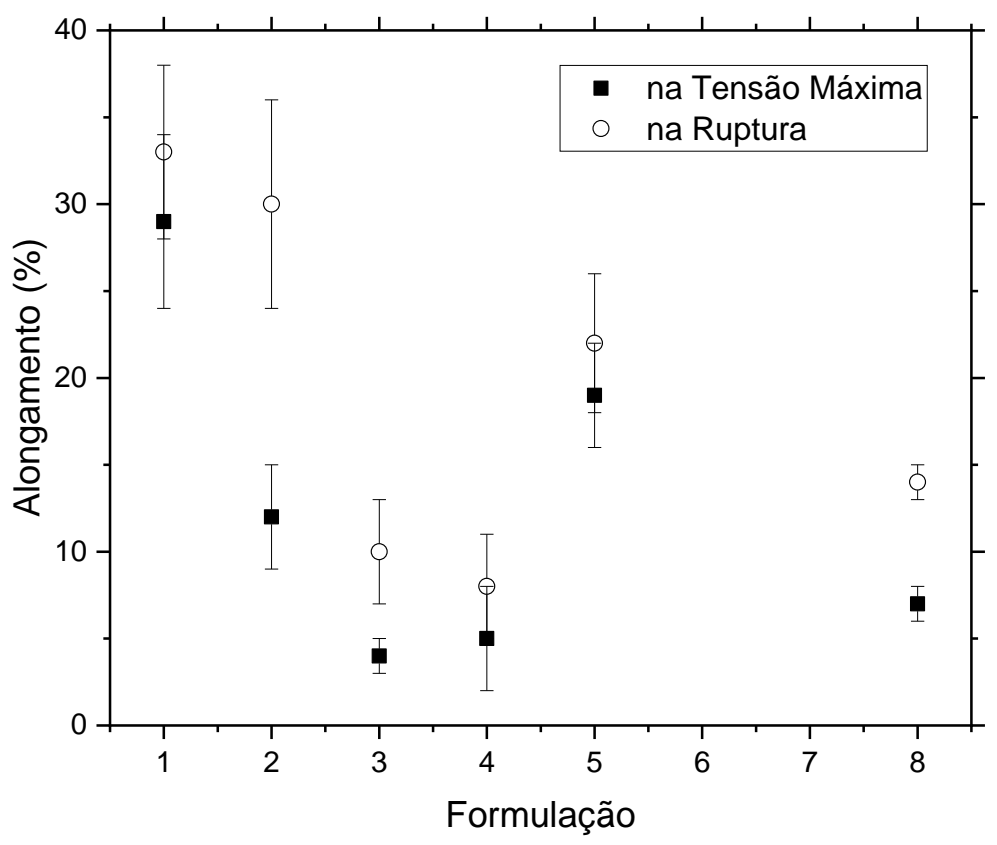

Figura 7: Alongamento na Tensão Máxima e na Ruptura (\%) por formulação de propelente curado.

As formulações 1, 3, 5 apresentaram valores de módulo de elasticidade similares. As formulações 2 e 8 apresentaram valores reduzidos de módulo de elasticidade, tensão máxima e tensão na ruptura. No caso da formulação 2, a presença de BDO em pequena quantidade funcionou como plastificante, tornando o material dúctil, porém com baixa resistência à tração, enquanto na formulação 80 alto teor de plastificante foi responsável por esta observação. A formulação 4 apresentou valor de módulo de elasticidade elevado, devido à elevada formação de ligações cruzadas pelo alto teor de BDO nesta formulação. A formulação 3 apresentou comportamento intermediário, em termos de módulo de elasticidade, entre as formulações 2 e 4 , como de fato era esperado, haja visto que o teor de BDO na formulação 3 está compreendido entre os teores existentes nas formulações 2 e 4 . Com relação à formulação 5, fica evidente que a presença de propilenoglicol, já a partir de menores quantidades, confere ao material maior módulo de elasticidade, se comparado com às formulações com BDO de mesmo teor. As formulações 6 e 7 não tiveram corpos de prova obtidos devido à alta viscosidade, no carregamento dos moldes, devido a um pot life reduzido, observado nas formulações com teor de propilenoglicol mais alto.

As tensões máximas e na ruptura para as formulações 2 e 8 foram menores, quando comparados as demais formulações, pelos motivos já apresentados no parágrafo anterior. O aumento no teor de diol, no caso do 1,4-butanodiol, acaba por aumentar os valores de tensão máxima e na ruptura, sendo maior para a formulação 4, com x igual a 0,15 . Os valores de tensão máxima e na ruptura para a formulação 5 , com x igual a 0,05 , empregando propilenoglicol, foram maiores que os observações na formulação 2 , também com $x$ igual a 0,05, mas empregando o BDO como diol.

Em relação aos alongamentos na tensão máxima e na ruptura, o aumento no teor de diol, no caso do 1,4-butanodiol, acaba por diminuir os valores de alongamento na tensão máxima e na ruptura, sendo menor para a formulação 4, com x igual a 0,15 . Do mesmo modo, os alongamentos na tensão máxima e na ruptura para a formulação 5, com x igual a 0,05, empregando propilenoglicol, foram maiores que as observações na formulação 2, com x igual a 0,05, com o emprego de BDO.

Ademais, ficou evidente que o elevado teor de plastificante na formulação 8, impediu que a cura fosse completa, demonstrado pelo baixo valor de módulo de elasticidade, tensões máxima e na ruptura. 


\subsubsection{Ensaio de raio-x}

As radiografias obtidas das amostras das formulações 1 a 8 são apresentadas na Figura 8, a seguir.

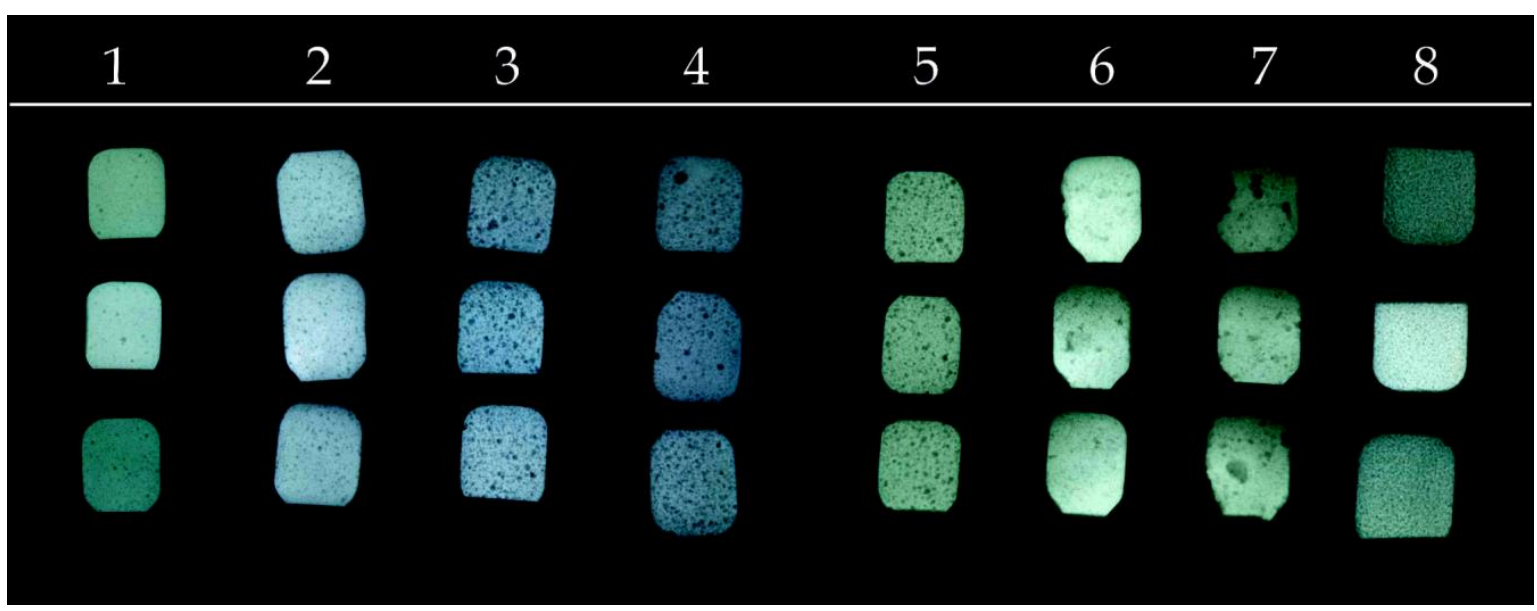

Figura 8. Radiografias obtidas das amostras das formulações 1 a 8 .

Verifica-se, a partir da Figura 8, uma tendência no aumento de bolhas, ou vazios, nas amostras conforme aumenta-se o teor de 1,4-butanodiol, visto que as formulações 3 e 4 , principalmente, apresentaram maior incidência de vazios na massa quando comparadas com a formulação 1 , sem a presença de BDO, e a formulação 2, que possui teor reduzido do mesmo. O 1,4-butanodiol é mais higroscópico que a resina. Neste sentido, o controle de umidade adotado no macerador, ao se acionar a linha de vácuo após a adição de cada componente da formulação e fechá-la em seguida, não se mostrou adequado em processamentos com BDO. Assim, é provável que a água absorvida reagiu com o IPDI, formando como subproduto da cura o gás carbônico, que resultou nos vazios apresentados.

Verifica-se também uma tendência no aumento de bolhas, ou vazios, nas amostras ao se adicionar 1,2propanodiol, mesmo em pequenos teores, visto que a formulação 5 apresenta maior incidência de vazios na radiografia quando comparada com a formulação 1 , sem a presença de propilenoglicol, e com a formulação 2, com mesmo teor de 1,4-butanodiol. O 1,2-propanodiol é mais higroscópico que a resina e que o BDO e, portanto, o controle de umidade adotado no macerador, já mencionado, também não se mostrou adequado em processamentos com o propilenoglicol. Assim, é provável que a água absorvida tenha reagido com o IPDI, que resultou nos vazios apresentados, na formulação 5. Com relação às formulações 6 e 7, estas massas não apresentaram fluidez no carregamento, de modo que os vazios apresentados nas radiografias são em função sobretudo da incapacidade das massas em se acomodarem, ou compactarem, no início da cura.

Por outro lado, comparando-se as radiografias das formulações 8 e 4, verificou-se menor incidência de vazios na formulação 8, que possuía maior teor de DOA. Este fenômeno pode ser explicado pela menor viscosidade desta massa, o que pode ter facilitado a saída das bolhas que porventura tenham sido geradas durante a cura, de modo que o plastificante além de facilitar o processamento e carregamento, evita que as possíveis bolhas geradas continuem no seio da massa. No entanto, as formulações com 1,4-butanodiol ao serem processadas sob vácuo durante toda a operação não produziriam propelentes com vazios ou bolhas, o que é fundamental para aplicações em foguetes espaciais.

\subsubsection{Determinação da massa específica do propelente}

Os resultados de determinação de massa específica dos distintos propelentes estudados são apresentados na Tabela 5. 
Tabela 5: Resultados de massa específica observados para as amostras de propelente curado.

\begin{tabular}{|c|c|c|c|c|}
\hline FORMULAÇÃO & DIOL & NOMENCLATURA & MASSA ESPECÍFICA $\left(\mathrm{g} \mathrm{cm}^{-3}\right)$ & $\mathrm{s}\left(\mathrm{g} \mathrm{cm}^{-3}\right)$ \\
\hline 1 & - & BDO/PPG $0 \%$ & 1,721 & 0,002 \\
\hline 2 & $\mathrm{BDO}$ & BDO $5 \%$ & 1,682 & 0,001 \\
\hline 7 & PPG & PPG $15 \%$ & 1,650 & 0,020 \\
\hline 5 & PPG & PPG $5 \%$ & 1,644 & 0,006 \\
\hline 4 & $\mathrm{BDO}$ & BDO $15 \%$ & 1,604 & 0,007 \\
\hline 8 & $\mathrm{BDO}$ & BDO $15 \%$ Modificada & 1,595 & 0,005 \\
\hline 6 & PPG & PPG $10 \%$ & 1,593 & 0,006 \\
\hline 3 & $\mathrm{BDO}$ & BDO $10 \%$ & 1,581 & 0,009 \\
\hline
\end{tabular}

Conforme comentado no item anterior, as massas 3, 4, 5, 6 e 7 apresentaram maiores incidências de vazios, enquanto a massa 1 a menor incidência de bolhas. Neste sentido, esperava-se que as formulações 3, 4, 5, 6 e 7 apresentassem valores de massa específica inferiores as demais, o que de fato foi verificado. A formulação 8 apresentou massa específica inferior a cinco das sete demais formulações. Este fato pode ser explicado pelo fato de a cura não ter sido plenamente obtida, visto que o processo de cura pode contrair a massa, aumentando sua densidade.

Desse modo, a determinação da massa específica, em conjunto com análise das radiografias do propelente são importantes na avaliação qualitativa da presença de vazios ou bolhas na formulação e avaliar o grau de cura do material.

\subsubsection{Bomba calorimétrica}

Os resultados obtidos nos ensaios de Bomba Calorimétrica, em atmosfera oxidante (combustão) e inerte (explosão) [30] são apresentados graficamente na Figura 9.

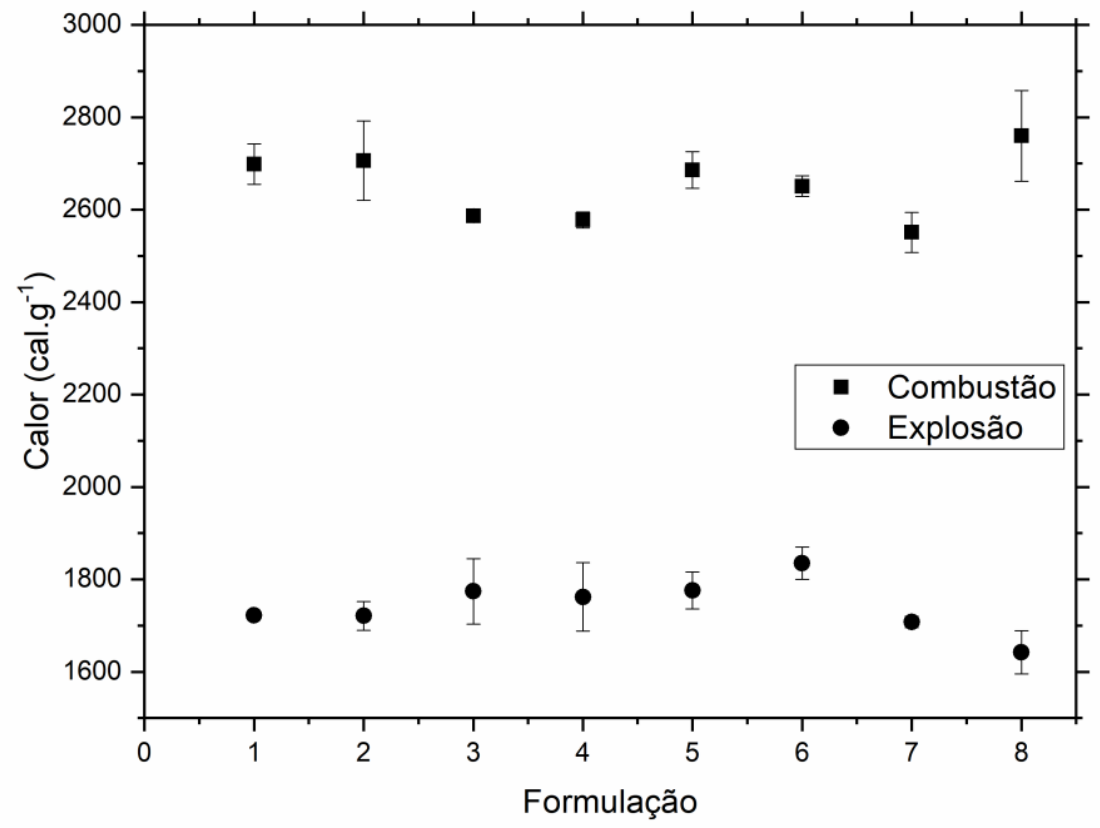

Figura 9: Resultados de calor de combustão e explosão obtidos por calorimetria.

Em primeiro lugar, os dados sugerem que, em princípio, a composição do binder não afetou consideravelmente os valores de energia liberados nos processos de combustão ou explosão das formulações estudadas. De fato, como sugerido na literatura [3, 7], o binder não tem como função preponderante aportar conteúdo energético aos propelentes. A função principal do binder é suportar as cargas sólidas que são 
adicionadas nas massas de propelentes, funcionando como aglutinante.

O teor de sólidos foi o mesmo para as formulações 1 a 7 , de modo que os valores de calor de combustão e explosão determinados deveriam ser similares para estas amostras. A formulação 8 apresentava menor teor de sólidos, ou cargas energéticas, em função da maior quantidade de DOA empregado na mesma. Portanto, esperava-se que os calores determinados para a formulação 8 fossem ligeiramente menores. No entanto, observou-se que o calor de combustão dessa formulação foi maior que das demais, contrapondo o resultado esperado. Ao mesmo tempo, o valor obtido de calor de explosão, determinado em atmosfera inerte, foi menor para a formulação 8 .

No estudo de propelentes, o calor de explosão é uma medida de energia liberada mais condizente às situações operacionais de voo, tendo em vista que a pressão de operação da câmara de queima é superior à pressão externa, impedindo a admissão de ar oxidante na mesma. Sendo assim, a análise do calor de explosão, cujos resultados obtidos estão alinhados aos resultados esperados, é mais útil que os dados de calor de combustão.

Há de se comentar também a diferença observada nos calores de explosão (atmosfera inerte) com os calores de combustão (atmosfera oxidante). No primeiro caso, as espécies oxidantes, para garantir a reação completa, está contida apenas na formulação, enquanto no segundo caso há espécies químicas oxidantes em excesso, pela presença de $\mathrm{O}_{2}$. Neste sentido, em função dos valores apresentados, diferentes entre si para mesma formulação, pode-se afirmar que não há o balanço estequiométrico correto e/ou ideal nas formulações estudadas, de forma a garantir a queima completa da formulação sob atmosfera inerte. A queima completa, ou com maior avanço, pode ter sido garantida sob atmosfera oxidante, em virtude de os valores de energia liberados terem sido maiores.

Assim, verificou-se, a partir dos dados de calor de explosão, que a presença de 1,4-butanodiol ou 1,2propanodiol, em diferentes quantidades, mantendo o teor de sólidos constante, não influenciou consideravelmente nos valores de calor de explosão determinados. Por outro lado, verificou-se uma ligeira queda nos valores de calor de explosão quando há uma redução nos percentuais mássicos de carga sólida energética, como sugerido nos resultados de calor de explosão apresentados pela formulação 8 .

\section{CONCLUSÕES}

O presente estudo teve como objetivo avaliar criteriosamente formulações completas de propelentes sólidos compósitos empregando dois diferentes tipos de dióis de cadeia curta, em diferentes quantidades.

Com relação aos aspectos operacionais dos processamentos, concluiu-se que a adição de dióis de cadeia curta altera consideravelmente a reologia da massa de propelente antes da adição do agente de cura. Concluiu-se também que, eventualmente, a adição de dióis no início do processo pode acarretar a perda por evaporação dos mesmos, de modo que foi sugerido a adição desses componentes no final do processo, antes da adição do agente de cura. Concluiu-se também que a reação de cura entre o propilenoglicol e o IPDI é muito rápida, de modo que nas formulações com maior teor deste diol não foi possível a obtenção de corpos de prova para ensaios de tração, visto que as massas 6 e 7 não apresentavam fluência no momento do carregamento. Neste sentido, o tempo de mistura após a adição de IPDI deveria ter sido reduzido.

Com relação aos processos de cura, concluiu-se que os dióis em baixos teores podem retardar o processo de cura, funcionando parcialmente como plastificante. Por outro lado, teores elevados, para $\mathrm{x}$ igual a 0,10 ou 0,15 , a cura foi mais acelerada, devido ao aumento mais rápido da dureza das amostras durante a cura.

Os ensaios de caracterização mecânica, dureza e tração, levaram a concluir que o aumento na quantidade de dióis eleva a dureza e o módulo de elasticidade das formulações. Além disso, esse efeito é mais evidente nas formulações com propilenoglicol, explicado pelo fato da molécula do mesmo ser menor, conferindo menor mobilidade à matriz polimérica. Neste sentido, em termos de propriedades mecânicas, as formulações com 1,4-butanodiol são mais promissoras, sendo necessário um ajuste fino dos valores de $\mathrm{x}$ e do teor de plastificante, que se mostrou elevado na formulação 8 .

A caracterização conjunta realizada pelos ensaios de Raio-X e determinação de massa específica foi útil na avaliação do grau de cura das formulações e do teor de vazios. Propelentes mais reticulados apresentaram maior valor de massa específica, devido à contração ocorrida no processo de cura. Por outro lado, o ensaio de Raio-X foi importante na avaliação da influência de dióis na formação de vazios no interior da 
massa, sendo verificado que a presença de dióis pode propiciar a formação de bolhas devido ao seu maior caráter higroscópico.

O ensaio de bomba calorimétrica permitiu concluir que modificações na formulação do binder não influenciam nos resultados de calor de combustão e explosão. Era de fato esperado, tendo em vista que a energia liberada na queima do propelente é proveniente principalmente das cargas sólidas da formulação. Já a adição de maior quantidade de plastificante reduziu o calor de explosão da formulação 8, pela redução do percentual de sólidos energéticos da mistura. Também se concluiu que na avaliação de propelentes, o calor de explosão apresenta resultados mais alinhados ao esperado em voos, em função de sua determinação ser realizada em atmosfera inerte.

\section{AGRADECIMENTOS}

Os autores gostariam de agradecer ao Instituto Tecnológico de Aeronáutica, ao Instituto de Aeronáutica e Espaço e a Agência Espacial Brasileira pelo suporte operacional e financeiro.

\section{BIBLIOGRAFIA}

[1] KHATOON, H., IQBAL, S., IRFAN, M., et al. Review on the Production, Properties and Applications of Non-Isocyanate Polyurethane: A Greener Perspective. Progress in Organic Coatings, v. 154, p. 106-124, 2021.

[2] SEKKAR, V., RAUNIJA, T.S.K. Hydroxyl-Terminated Polybutadiene-Based Polyurethane Networks as Solid Propellant Binder-State of the Art. Journal of Propulsion and Power, v. 31, v. 1, p. 16-35, 2015.

[3] CHATURVEDI, S., DAVE, P.N. Solid Propellants: AP/HTPB Composite Propellants. Arabian Journal of Chemistry, v. 12, v. 8, p. 2061-2068, 2019.

[4] GHOSH, K., GAIKWAD, L.V., KALAL, R.K., et al. Light Weight HTPB-Clay Nanocomposites (HCN) with Enhanced Ablation Performance as Inhibition Materials for Composite Propellant. Defence Technology, v. 17, n. 2, p. 559-570, 2021.

[5] SUTTON, G.P., BIBLARZ, O. Rocket propulsion elements, 7. ed, New York, NY, Wiley-Interscience, 2001.

[6] VARGHESE, T.L., KRISHNAMURTHY, V.N., The Chemistry and Technology of Solid Rocket Propellants (A Treatise on Solid Propellants). New Dheli, Allied Publishers PVT. LTD, 2017.

[7] DAVENAS, A. Solid Rocket Propulsion Technology, 1. ed, Oxford, UK, Pergamon Press, 1993.

[8] SCHLINGLOFF, H. Astronautical Engineering. Kalkofenring, Bad Abbach, 2005.

[9] WILliamS, F.A., BARRERE, M., HUANG, M.C. Fundamental Aspects of Solid Propellant Rockets. Slough, England, Technivision Services, 1989.

[10] AGRAWAL, J.P. High Energy Materials: Propellants, Explosives and Pyrotechnics., Weinheim, WileyVCH, 2010.

[11] PIMONT, L.J., FERNANDES, P.C.G., FERRÃO, L.F.A., et al. Study on the Mechanical Properties of Solid Composite Propellant Used as a Gas Generator. Journal of Aerospace Technology and Management, v. 11, n. 1, p. 7-10, 2020.

[12] AZIZ, A., MAMAT, R., WAN ALI, W.K., et al. Review on Typical Ingredients for Ammonium Perchlorate Based Solid Propellant. Applied Mechanics and Materials, v. 773-774, n. 15, p. 470-475, 2015.

[13] ADEL, W.M., LIANG, G. Service Life Prediction of AP/A1/HTPB Solid Rocket Propellant with Consideration of Softening Aging Behavior. Chinese Journal of Aeronautics, v. 32, n. 2, p. 361-368, 2019.

[14] FUENTE, J.L. LA, RODRÍGUEZ, O. Dynamic Mechanical Study on the Thermal Aging of a HydroxylTerminated Polybutadiene-Based Energetic Composite. Journal of Applied Polymer Science, v. 87, n. 14, p. 2397-2405, 2003.

[15] KIM, S.-H., IM, Y.-T. Experimental Study of Material Behavior of AP-HTPB Base Composite Solid Propellant. Journal of Mechanical Science and Technology, v. 33, n. 7, p. 3355-3361, 2019.

[16] O’NEIL, P.T., HEISTER, S.D. Evaluation of Processing and Mechanical Parameters of Dicyclopentadiene-Based Gumstocks. Journal of Propulsion and Power, v. 29, n. 6, p. 1311-1322, 2013. 
[17] VERMA, S., RAMAKRISHNA, P.A. Effect of Mixer Size on Density and Burn Rate of Composite Solid Propellant. In: Anais... 47th AIAA/ASME/SAE/ASEE Joint Propulsion Conference and Exhibit, pp. 19, August, 2011.

[18] AMIN, N., SIDIG, B., ABUUZNIEN, M. Feasibility Of Extending The Composite Propellant Pot Life. In: Anais... The International Conference on Chemical and Environmental Engineering, p. 1-7, 2012.

[19] MAHANTA, A.K., GOYAL, M., PATHAK, D.D. Empirical Modeling of Chemoviscosity of Hydroxy Terminated Polybutadiene Based Solid Composite Propellant Slurry. Malaysian Polymer Journal, v. 5, n. 1, 2010.

[20] JAIN, M., SINGH, D., MEHILAL, BALASUBRAMANIAN, K., et al. Studies on Prediction of Rheological Behavior of Composite Propellant Formulations Based on Rheological Models. Advanced Science, Engineering and Medicine, v. 7, n. 8, p. 690-696, 2015.

[21] IQBAL, M.M., RAZA CH., S., LIANG, W., et al. Investigating The Effect Of Solid Fillers On Mechanical And Rheological Properties Of Composite Propellants. International Journal of Energetic Materials and Chemical Propulsion, v. 12, n. 1, p. 75-86, 2013.

[22] VELLAISAMY, U., BISWAS, S. Effect of Metal Additives on Neutralization and Characteristics of AP/HTPB Solid Propellants. Combustion and Flame, v. 221, p. 326-337, 2020.

[23] SURESH BABU, K.V., KANAKA RAJU, P., THOMAS, C.R., et al. Studies on Composite Solid Propellant with Tri-Modal Ammonium Perchlorate Containing an Ultrafine Fraction. Defence Technology, v. 13, n. 4, p. 239-245, 2017.

[24] ZHANG, L., LIN, Q., CHENG, B., et al. Effect of Hexanitroethane (HNE) and Hydrazinium Nitroformate (HNF) on Energy Characteristics of Composite Solid Propellants. FirePhysChem, v. 1, n. 2, p. 116-122, 2021.

[25] DILliER, C.A.M., PETERSEN, E.D., PETERSEN, E.L. Isolating the Effects of Oxidizer Characteristics and Catalytic Additives on the High-Pressure Exponent Break of AP/HTPB-Composite Propellants. Proceedings of the Combustion Institute, v. 38, n. 3, p. 4409-4416, 2021.

[26] RAO, B.N., MALKAPPA, K., KUMAR, N., et al. Ferrocene Grafted Hydroxyl Terminated Polybutadiene: A Binder for Propellant with Improved Burn Rate. Polymer, v. 63, p. 162-170, 2019.

[27] MCCLAIN, M.S., GUNDUZ, I.E., SON, S.F. Additive Manufacturing of Ammonium Perchlorate Composite Propellant with High Solids Loadings. Proceedings of the Combustion Institute, v. 37, n. 3, p. 3135-3142, 2019.

[28] SHEIKHY, H., SHAHIDZADEH, M., RAMEZANZADEH, B., et al. Studying the Effects of Chain Extenders Chemical Structures on the Adhesion and Mechanical Properties of a Polyurethane Adhesive. Journal of Industrial and Engineering Chemistry, v. 19, n. 6, p. 1949-1955, 2013.

[29] WINGBORG, N. Increasing the Tensile Strength of HTPB with Different Isocyanates and Chain Extenders. Polymer Testing, v. 21, n. 3, p. 283-287, 2002.

[30] FIFER, R.A., MORRIS, J.B. Techniques for the Estimation of Heats of Explosion (HEX) Using Thermochemical Codes. [s.l: s.n.].

\section{ORCID}

Bruno Cesar Christo da Cunha

José Atílio Fritz Fidel Rocco http://orcid.org/0000-0002-6763-3596

http://orcid.org/0000-0002-6004-6997 